TITLE:

\title{
Fluorous Property of a Short Perfluoroalkyl-Containing Compound Realized by Self- Assembled Monolayer Technique on a Silicon Substrate
}

\section{AUTHOR(S):}

Kise, Ryuma; Fukumi, Aki; Shioya, Nobutaka; Shimoaka, Takafumi; Sonoyama, Masashi; Amii, Hideki; Takagi, Toshiyuki; Kanamori, Toshiyuki; Eda, Kazuo; Hasegawa, Takeshi

\section{CITATION:}

Kise, Ryuma ...[et al]. Fluo rous Property of a Short Perfluoroalkyl-Containing Compound Realized by Self-Assembled Monolayer Technique on a Silicon Substrate. Bulletin of the Chemical Society of Japan 2019, 92(4): 785-789

\section{ISSUE DATE:}

2019-01-19

\section{URL:}

http://hdl.handle.net/2433/241217

\section{RIGHT:}

(C) 2019 The Chemical Society of Japan; 発行元の許可を得て登録してい ます.; This is not the published version. Please cite only the published version.; この論文は出版社版でありません。引用の際には出版社版を ご確認ご利用ください。 


\title{
Fluorous Property of a Short Perfluoroalkyl-Containing Compound Realized by Self- Assembled Monolayer Technique on a Silicon Substrate
}

\author{
Ryuma Kise', Aki Fukumi ${ }^{1}$, Nobutaka Shioya ${ }^{1}$, Takafumi Shimoaka ${ }^{1}$, Masashi Sonoyama ${ }^{2}$, Hideki Amii ${ }^{2}$, Toshiyuki \\ Takagi $^{3}$, Toshiyuki Kanamori ${ }^{3}$, Kazuo Eda ${ }^{4}$ and Takeshi Hasegawa ${ }^{1 *}$ \\ ${ }^{1}$ Institute for Chemical Research, Kyoto University, Gokasho, Uji, Kyoto 611-0011, Japan \\ ${ }^{2}$ Division of Molecular Science, Faculty of Science and Technology, Gunma University, Kiryu, Gunma 376-8515. , Japan \\ ${ }^{3}$ National Institute of Advanced Industrial Science and Technology, Tsukuba Central 5, 1-1-1 Higashi, Tsukuba, Ibaraki 305-8565, Japan
}

${ }^{4}$ Department of Chemistry, Graduate School of Science, Kobe University, 1-1 Rokko-dai, Nada-ku, Kobe, Hyogo 657-8501, Japan

E-mail: htakeshi@scl.kyoto-u.ac.jp

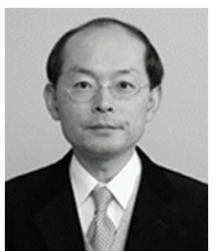

\section{Takeshi Hasegawa}

Takeshi Hasegawa received PhD from Kyoto University. He was appointed to be Assistant Professor at Kobe Pharmaceutical University in 1993 and Lecturer in 2001. After he experienced Associate Professor at CIT, Nihon University (2003), Researcher of JST PRESTO (2004), and Associate Professor at Tokyo Institute of Technology (2006), he joined ICR, Kyoto University as Full Professor in 2011. He is a Fellow of Society for Applied Spectroscopy (USA) after recognition of his original spectroscopic technique of "pMAIRS." The SDA theory is another his exciting topic.

\begin{abstract}
Fluorous properties represented by the water-and-oil repellency are perfluoroalkyl $\left(\mathrm{R}_{\mathrm{f}}\right)$ compounds-specific characters, which are widely used for surface coating of glass, an electronic device and textiles for preventing water and greasy fouling. According to the stratified dipole-arrays (SDA) theory, the minimum $\mathrm{R}_{\mathrm{f}}$ length of $\left(\mathrm{CF}_{2}\right)_{7}$ is theoretically necessary for realizing the fluorous property. Unfortunately, however, compounds involving this chemical unit are strictly banned for fabrication because of a concern of environmental pollution, which is a big dilemma. Here, we show that the fluorous property can be realized by the self-assembled monolayer (SAM) technique even with a short $\mathrm{R}_{\mathrm{f}}$-containing compound, since the SAM technique makes the best use of the self-aggregation property of the $R_{f}$ groups, and it readily makes the molecules immobile.
\end{abstract}

Keywords: Short perfluoroalkyl-containing compound, Self-assembled monolayer technique, Fluorous surface property

\section{Introduction}

Perfluoroalkyl $\left(\mathrm{R}_{\mathrm{f}}\right)$ compounds are characterized by the unique "fluorous" properties represented by the water-and-oil repellency, which cannot be realized by non-fluorine compounds. ${ }^{1}$ The fluorous property is strongly correlated with the molecular interaction between the $\mathrm{R}_{\mathrm{f}}$ chains. ${ }^{2}$ For many years, this has often been discussed on London's dispersion force, as commonly found in normal hydrocarbon compounds, which is, unfortunately, an obstacle for comprehensive understanding of the material properties of $\mathrm{R}_{\mathrm{f}}$ compounds. ${ }^{2,3}$

To discuss the molecular interaction of $\mathrm{R}_{\mathrm{f}}$ groups in a physicochemical manner, we have to pay attention that each $\mathrm{C}-$ $\mathrm{F}$ bond has a large permanent dipole moment due to the large electronegativity of fluorine $\mathrm{e}^{3}$, and in addition, an $\mathrm{R}_{\mathrm{f}}$ group has a helical conformation about the molecular axis. ${ }^{4,5}$ As a result, the $\mathrm{R}_{\mathrm{f}}$ groups having the length of $\left(\mathrm{CF}_{2}\right)_{7}$ or longer are spontaneously aggregated to yield "two-dimensional dipolearrays with various directions," which is a dipole-array network. This spontaneous aggregation mechanism is known to be the stratified dipole-arrays (SDA) theory. ${ }^{2,6}$ The comprehensive understanding of the fluorous properties fundamentally requires the SDA theory; whereas the hydrophobicity is partly attributed to the surface morphology. ${ }^{7}$

On the SDA theory, the $\mathrm{R}_{\mathrm{f}}$ length of $\left(\mathrm{CF}_{2}\right)_{7}$ is the critical minimal length for inducing the 'spontaneous' aggregation, and the fluorous property is, in fact, induced by the molecular aggregate of $\left(\mathrm{CF}_{2}\right)_{7}$ or longer. ${ }^{1,2,6}$ This boundary length is often found in various compounds, and poly(fluoroacrylate) (PFA) is one of the good examples. PFA has side chains, each of which has an $\mathrm{R}_{\mathrm{f}}$ substitute. If the substitute is the perfluorooctyl group (i.e., $\left.-\left(\mathrm{CF}_{2}\right)_{7} \mathrm{CF}_{3}\right)$ or longer, PFA exhibits an apparent fluorous property; whereas the property is lost for a shorter $\mathrm{R}_{\mathrm{f}}$ substitute. ${ }^{8}$ This critical length of $\left(\mathrm{CF}_{2}\right)_{7}$ works as a key not only for a useful material function, but also for a troubling property.

PFOX represented by perfluorooctyl sulfonate (PFOS) and perfluorooctanoic acid (PFOA) has recently been pointed out to be troubling: they are toxic and persist in environment for a longterm period. ${ }^{9}$ The same problem is true of a longer $\mathrm{R}_{\mathrm{f}}$ compound. The critical chemical group is the perfluorooctyl group that involves the unit of $\left(\mathrm{CF}_{2}\right)_{7}$. On the SDA theory, these compounds would spontaneously make a molecular aggregate, and the tightly packed aggregate should be inappropriate for smooth metabolism, once they are uptaken into a human body. Therefore, PFOX or longer relatives are banned to be manufactured on considering the environmental pollution; whereas the critical length of $\left(\mathrm{CF}_{2}\right)_{7}$ is necessary for realizing the useful fluorous properties, ${ }^{8}$ which is a big dilemma. A new technical recipe based on a comprehensive theory is therefore strongly needed for realizing the fluorous property using a short $\mathrm{R}_{\mathrm{f}}$-containing compound having $\left(\mathrm{CF}_{2}\right)_{6}$ or shorter.

Fortunately, we have an opportunity to reconsider a point of the SDA theory that the molecules are "spontaneously" aggregated for the length of $\left(\mathrm{CF}_{2}\right)_{7}$ or longer. If we could "artificially arrange" short $\mathrm{R}_{\mathrm{f}}$ groups closely in a twodimensional (2D) manner, the surface property of the membrane is expected to be close to that obtained by PFOX. ${ }^{3}$

In the present study, a silicon ( $\mathrm{Si}$ ) substrate is chosen as the substrate, and artificially arranged molecular aggregates are prepared by using the Langmuir-Blodgett $(\mathrm{LB})^{10}$ and selfassembled monolayer (SAM) techniques. ${ }^{11-18}$ Since a SAM 
coverage is attained by controlling the surface reactivity with the substrate surface, SAM is an artificial molecular aggregate. As a result, very low surface energy is readily realized even for a short- $\mathrm{R}_{\mathrm{f}}$ containing compound by the SAM technique; whereas the LB technique is inadequate for the stability of the hydrophobicity. In addition, the SAM technique is found to retain a very low surface energy for a long time without using PFOX.

\section{Experimental}

Chemicals. $1 H, 1 H, 2 H, 2 H$-Perfluorododecyltrichlorosilane (FDDTS; $\left.\mathrm{R}_{\mathrm{f}} 9\right) \quad(97 \%)$ and $1 \mathrm{H}, 1 \mathrm{H}, 2 \mathrm{H}, 2 \mathrm{H}$-perfluorooctyltrichlorosilane (FOTS; Rf5), (97\%) were purchased from SigmaAldrich (St. Louis, MO, USA). 1H,1H,2H,2H-perfluorodecyltrichlorosilane (FDTS; Rf7) $(>96.0 \%)$ was purchased from Tokyo Chemical Industry Co. Ltd. (Tokyo, Japan). $5,5,6,6,7,7,8,8,9,9,10,10,11,11,12,12,13,13,14,14,14$-Henicosafluorotetradecanoic acid (My- $\left.\mathrm{R}_{\mathrm{f}} 9\right)$ for the discussion of the LB technique was synthesized as reported in our previous paper. ${ }^{6}$

The Si substrate was purchased from Valqua FFT Inc. (Tokyo, Japan) and cleaned in a conventional manner: sonication in pure water, ethanol, acetone, and dichloroethane for about 1 min each. All the organic solvents were guaranteed reagents. The substrate was oxidized using a Bio Force (Ames, IA) PC440 UV/ozone ProCleaner ${ }^{\mathrm{TM}}$ Plus for $10 \mathrm{~min}$.

Langmuir-Blodgett (LB) Film. A Langmuir (L) film was prepared on pure water by spreading a solution of $M y-R_{f} 9$, which was dissolved in a mixed solvent of methanol and chloroform (1 : $10(\mathrm{v} / \mathrm{v}))$, and the $\mathrm{L}$ film of a single monolayer was transferred onto a Si substrate by using the LB technique with a transfer ratio of 1.02. To prepare the LB film of CdMy-Rf9, a buffered aqueous solution of cadmium chloride $\left(\mathrm{CdCl}_{2}\right)\left(1.0 \times 10^{-}\right.$ $\left.{ }^{2} \mathrm{M}\right)$ was used as the subphase, in which sodium hydrogen carbonate $\left(\mathrm{NaHCO}_{3}\right)$ and sodium carbonate $\left(\mathrm{Na}_{2} \mathrm{CO}_{3}\right)$ were added to keep $\mathrm{pH} \approx 7.5 .{ }^{19} \mathrm{CdCl}_{2}$ was purchased from Rare Metallic Co. Ltd. (Tokyo, Japan), and the other chemicals were purchased from Wako Pure Chemical Industries, Ltd. (Osaka, Japan).

The LB films were prepared by using a Biolin Scientific (Espoo, Finland) KSV-NIMA Minitrough Langmuir-Blodgett system. Pure water for subphase was obtained by a Millipore (Molsheim, France) Elix UV-3 pure-water generator and Symplicity UV System water purifier. The water exhibited an electric resistivity higher than $18.2 \mathrm{M} \Omega \mathrm{cm}$.

SAM Preparation. SAM reagents were dissolved in a fluorine containing solvent, HCFC-225 (1.0 mM), purchased from Wako Pure Chemical Industries, Ltd. (Osaka, Japan), and a Si substrate was dipped in the solution for $60 \mathrm{~min}$. To promote the SAM formation, the solution was kept at $70^{\circ} \mathrm{C}$. Unreacted molecules remained on the SAM were washed away by ASAHIKLIN AK225AES, made by Asahi Glass Co., Ltd. (Tokyo, Japan), and the solvent remained was removed in a dry oven at $60^{\circ} \mathrm{C}$.

Contact Angle Measurements. Contact angle was measured using a Kyowa Interface Science Co., Ltd. (Saitama, Japan) DropMaster, DM-501Hy, contact-angle meter, with which the surface tension of pure water $\left(72.5 \mathrm{mN} \mathrm{m}^{-1}\right)$ at $25{ }^{\circ} \mathrm{C}$ was checked. The drop of water (or diiodomethane $\left(\mathrm{CH}_{2} \mathrm{I}_{2}\right)$ purchased from Wako Pure Chemical Industries, Ltd. (Osaka, Japan)) with a volume of $2.0 \mu \mathrm{L}$ was put on the sample surface, and the static contact angle was estimated. Time-dependent contact angle was measured every one second.

Grazing Incidence X-ray Diffraction (GIXD) Measurements. GIXD measurements were carried out on a high-resolution Xray diffractometer (Rigaku SuperLab) equipped with a parabolic graded multilayer mirror. The $\mathrm{X}$-ray was generated by using a $\mathrm{Cu}$ rotating anode generator $(\mathrm{Cu} \mathrm{K} \alpha)$ operated at $40 \mathrm{kV}$ and 30
mA. The scattered X-ray was collected by a scintillation counter. The diffraction pattern was measured with an in-plane geometry, with which the angle of incidence was fixed at $0.2^{\circ}$. The scan speed was $0.2^{\circ} \mathrm{min}^{-1}$ and the sampling step was $0.03^{\circ}$.

\section{Results and Discussion}

LB-Film Approach. The LB technique is employed for preparing artificially aggregated molecules as a monolayer on a silicon substrate. An LB film of My-Rf 9 prepared at a surface pressure $(\pi)$ in the solid-film region $\left(35 \mathrm{mN} \mathrm{m}^{-1}\right.$; Figure $\left.\mathrm{S} 1\right)$ was, however, very unstable and it was soon removed simply by putting a water droplet on the monolayer for a contact angle measurement as found in Figure 1a. In fact, the contact angle of this sample is the same as that on a bare surface of $\mathrm{Si}$, which straightforwardly implies the removal of the LB film. The removal was confirmed by measurement of an IR spectrum (data not shown).
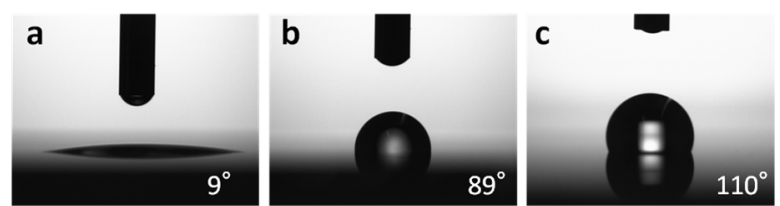

Figure 1. Contact-angle using a probe of a water droplet on (a) LB film of My-R $\mathrm{R}_{\mathrm{f}}$, (b) LB film of CdMy-R 9 and (c) SAM-R $\mathrm{f}$, each of which is prepared on $\mathrm{Si}$.

The unstableness of the monolayer on the contact with a water droplet should be attributed to the hydrophilic property of the carboxylic group. In short, the monolayer was soon dissolved in water.

To avoid the hydrophilicity, a robust LB film was prepared from a monolayer $\left(\pi=35 \mathrm{mN} \mathrm{m}^{-1}\right)$ on a cadmium chloride aqueous solution with a concentration of $1.0 \times 10^{-2} \mathrm{M}$ at $\mathrm{pH} 7.5^{19}$ to make the monolayer a cadmium (Cd) "salt" of My- $\mathrm{R}_{\mathrm{f}}$ 9

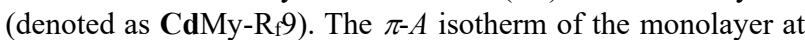
$25^{\circ} \mathrm{C}$ is available in Figure $\mathrm{S} 1$. Since the Coulomb interaction between the carboxylate anion and $\mathrm{Cd}^{2+}$ is strong, the monolayer of the Cd salt is known to be stiff. ${ }^{19}$ In this manner, a highly packed molecular aggregate is obtained with an aid of both monolayer compression and chemical aggregation by $\mathrm{Cd}^{2+}$. As expected, this LB film was not removed by the water-droplet probe.

Contrary to our expectation, however, the contact angle of the LB film of CdMy- $\mathrm{R}_{\mathrm{f}} 9$ is $89^{\circ}$ as shown in Figure 1a, which is apparently less than that of the reference data $\left(\mathrm{ca} .119^{\circ}\right)$ of a thick layer $(100 \mathrm{~nm})$ of $\mathrm{C}_{20} \mathrm{~F}_{42} .{ }^{20}$ Then, the same $\mathrm{LB}$ film is analyzed by GIXD measurements (Figure 2a). The GIXD patterns show a single peak at about $2 \theta=17.7^{\circ}$ reflecting a hexagonal crystalline packing, ${ }^{21,22}$ and its interplanar spacing $(d=0.4996 \mathrm{~nm})$ is apparently larger than that of the reference data, i.e., $0.4936 \mathrm{~nm}$ of $\mathrm{C}_{20} \mathrm{~F}_{42}$ calculated from the crystal data. ${ }^{21}$ This indicates that the molecular packing is not good enough even at the high surface pressure of $35 \mathrm{mN} \mathrm{m}^{-1}$.

The correlation between the molecular packing and the surface pressure is presented in Figure 2a d. The molecular packing is, as expected, systematically becoming looser on a decrease of the surface pressure. These changes are directly reflected by the contact angle as presented in Figure 2: the surface pressures of $35,25,15$ and $5 \mathrm{mN} \mathrm{m}^{-1}$ correspond to the contact angles of $89^{\circ}$, $78^{\circ}, 64^{\circ}$ and $42^{\circ}$, respectively, in a decreasing manner. This good correlation is understandable on the SDA theory. These results, therefore, imply that the molecular aggregation is a key for aiming at a high hydrophobicity using a short $\mathrm{R}_{\mathrm{f}}$ compound. 
The time-duration of the contact angle is of another interest. Figure 3 presents time courses of the contact angle measured on different two surfaces. The curve of the LB film of CdMy- $\mathrm{R}_{\mathrm{f}} 9$ is shown in Figure 3a. This curve is impressive that the hydrophobicity is quickly lost especially in the very early stage within $30 \mathrm{sec}$. When compared to the criteria line of $119^{\circ}$ (dashed line), the instability of the LB film is very apparent.

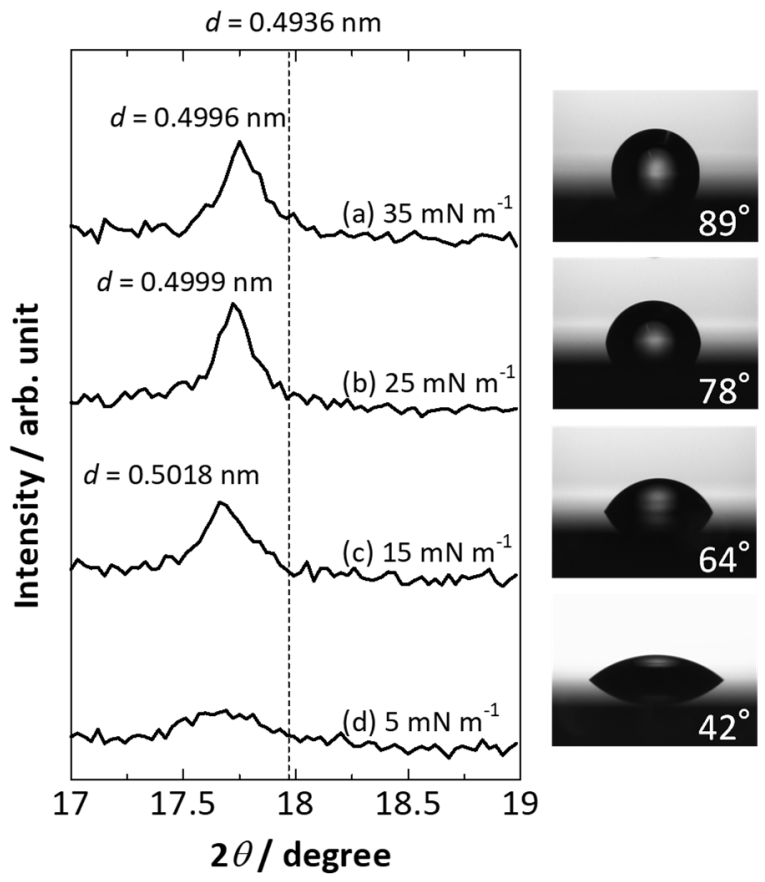

Figure 2. XRD (GIXD-IP) patterns of (a) a PTFE tape, and (b) an LB film of CdMy-R $\mathrm{R}_{\mathrm{f}} 9$ deposited at 35, (c) 25, (d) 15 and (e) $5 \mathrm{mN} \mathrm{m}^{-1}$ on a Si wafer.

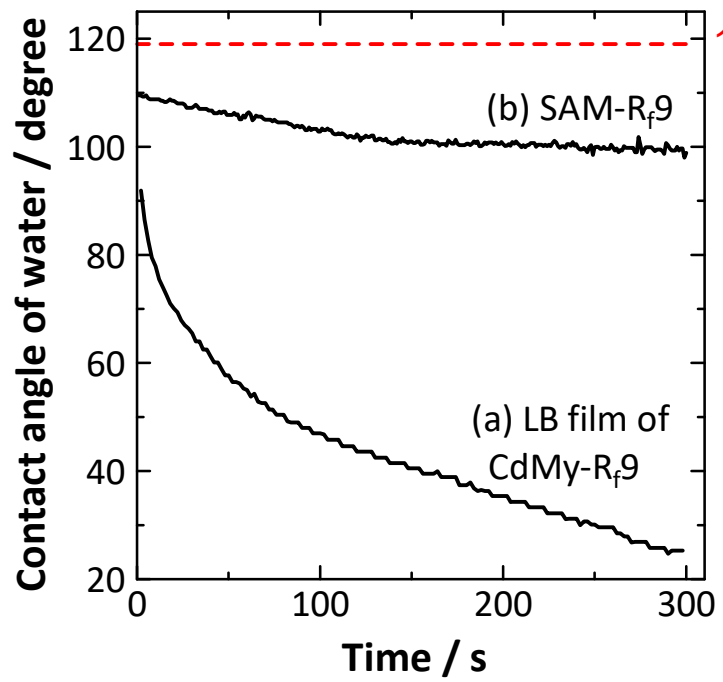

$119^{\circ}$

Figure 3. The time-courses of the contact angle measured on (a) the LB film of CdMy-R $\mathrm{R}_{\mathrm{f}}$, and (b) SAM- $\mathrm{R}_{\mathrm{f}} 9$.

My- $\mathrm{R}_{\mathrm{f}} 9$ is indeed stabilized by $\mathrm{Cd}^{2+}$ to be CdMy- $\mathrm{R}_{\mathrm{f}} 9$, which works well for protecting the head group from the hydration when a water droplet is put on the LB film. Nevertheless, the contact of the head group with the substrate surface is made weaker by losing the hydroxyl group $\left((\mathrm{R}-\mathrm{COO}){ }_{2} \mathrm{Cd}\right),{ }^{19}$ since the metal salt cannot actively be interacted with the oxide surface via the hydrogen bonding. This weak interaction between the LB film and the substrate should be an unstable factor of the 'molecular mobility' especially after putting a water droplet on it.

In this manner, for both cases of acid and salt, the LB technique is found not suitable for having a durable hydrophobic coating on a solid surface for a long time. In addition, the LB technique is not good for molecular packing, either, as discussed on Figure 2. As a conclusion, the best use of "spontaneous molecular aggregation" is necessary for a high hydrophobicity, and in addition the molecules should be immobile for a stable duration.

SAM Approach. To satisfy these two points simultaneously, the SAM technique is employed. Making a highly condensed and stable SAM on a Si surface is a little bit difficult task for normal hydrocarbons. ${ }^{12-17}$ The SAM formation requires nucleation of molecules at the initial stage, and the nucleus is developed by connecting with each other two-dimensionally. ${ }^{16}$ Very fortunately, $\mathrm{R}_{\mathrm{f}}$ groups intrinsically have a nucleation property due to the SDA packing, which is quite suitable for the SAM formation. ${ }^{2}$ In fact, the SAM of a silane-coupling reagent having an Rf9 group (FDDTS) was readily prepared in only 10 min. Both IR spectrum and GIXD-IP pattern (Figure S2) of the SAM apparently indicate that the SAM is ideally prepared in terms of molecular density and packing. In fact, as a result, the contact angle of the surface was $110^{\circ}$ as found in Figure 1c. In addition, the time-duration is largely improved as found in Figure 3b: the decrease of the contact angle is stopped at about $100^{\circ}$ that is good enough for the hydrophobicity.

In this manner, the SAM technique has proved to work for obtaining a highly durable hydrophobicity. To discuss the fluorous property, the surface free energy of SAM-Rf 9 was measured by employing the add-and-remove volume method, and a very small value of $11.1 \mathrm{~mJ} \mathrm{~m}^{-2}$ was obtained as found in Table $1 . .^{23}$ As a reference, relevant results of SAM of octadecyltrimethoxysilane (SAM-ODS; normal alkyl) are also presented in the table, which are also results of the present study. The value of $11.1 \mathrm{~mJ} \mathrm{~m}^{-2}$ is apparently smaller than the hydrocarbon $\left(24.7 \mathrm{~mJ} \mathrm{~m}^{-2}\right)$, which indicates that the surface of SAM- $\mathrm{R}_{\mathrm{f}} 9$ is fluorous. Even if this value is compared to the top record $\left(6.7 \mathrm{~mJ} \mathrm{~m}^{-2}\right)$ on a bulk material $\left(\mathrm{C}_{20} \mathrm{~F}_{42}\right.$ with thickness of $100 \mathrm{~nm}$ ) by Nishino et al., ${ }^{20}$ the free energy on the SAM can reasonably be recognized to be fluorous. Since the present result of a monolayer is comparable to the thick film, the SAM technique is found powerful for making the surface fluorous.

Table 1. Surface free energy of SAM-R $n$ determined by the Owens method

\begin{tabular}{|c|c|c|c|c|c|}
\hline & \multicolumn{2}{|c|}{$\mathrm{CA} /{ }^{\circ}$} & \multicolumn{3}{|c|}{ Surf. Free Energy $/ \mathrm{mJ} \mathrm{m}^{-2}$} \\
\hline & Water & $\mathrm{CH}_{2} \mathrm{I}_{2}$ & $\gamma_{\mathrm{s}}^{\mathrm{d}}$ & $\gamma_{\mathrm{s}}^{\mathrm{p}}$ & $\gamma$ \\
\hline SAM-Rf 5 & 106.7 & 88.8 & 12.0 & 1.9 & 13.9 \\
\hline SAM-R 7 & 108.9 & 90.0 & 11.7 & 1.5 & 13.2 \\
\hline SAM-R 9 & 112.4 & 94.6 & 9.9 & 1.2 & 11.1 \\
\hline $\mathrm{C}_{20} \mathrm{~F}_{42}{ }^{20}$ & 119 & 107 & 5.5 & 1.2 & 6.7 \\
\hline SAM-ODS & 107.0 & 67.0 & 24.6 & 0.1 & 24.7 \\
\hline
\end{tabular}

The dispersion and the polar components of the surface free energy $\left(\gamma_{\mathrm{s}}^{\mathrm{d}}\right.$ and $\gamma_{\mathrm{s}}^{\mathrm{p}}$, respectively) are both obtained by the Owens method using water and $\mathrm{CH}_{2} \mathrm{I}_{2}$ droplets (Table 1). Since the compound of SAM-R $n$ has a primary structure of $\mathrm{CF}_{3}\left(\mathrm{CF}_{2}\right)_{n}\left(\mathrm{CH}_{2}\right)_{2} \mathrm{SiCl}_{3}$, the relative portion of the normal hydrocarbon part, i.e., $\left(\mathrm{CH}_{2}\right)_{2}$, increases on a decrease of $n$, which induces the contribution of the "dispersion" part. ${ }^{2,3}$ In fact, $\gamma_{\mathrm{s}}^{\mathrm{d}}$ increases from 9.9 to $12.0 \mathrm{~mJ} \mathrm{~m}^{-2}$ systematically. At the same time, $\gamma_{s}{ }^{p}$ also increases slightly, which is because the summation of the dipoles of a short single $\mathrm{R}_{\mathrm{f}}$ group cannot be close to zero, and even a collection of the molecules would not be close to zero, 
either. ${ }^{2,3}$

As expected on the SDA theory, a surprisingly low surface energy is obtained for the short compound. This means that the fluorous property can be realized even with a short- $R_{f}$ chain containing compound by employing the SAM technique, which readily overcomes the "dilemma."

The time-duration is also found excellent for the short- $\mathrm{R}_{\mathrm{f}}$ compounds. The time-dependent static contact angles are presented in Figure 4. As found in this figure, the degradation is not significant especially for $R_{f} 7$. The contact angle of $R_{f} 5$ is still decreasing with time, which should be correlated with the decrease of the receding contact angle of the dynamic measurements (data not shown).
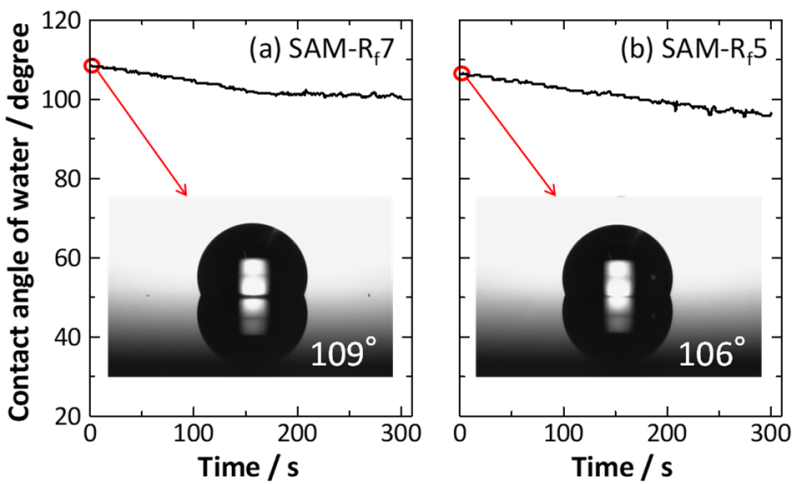

Figure 4. Time-dependent static contact angle on (a) SAM-R 7 and (b) $-\mathrm{R}_{\mathrm{f}} 5$ on $\mathrm{Si}$ at ambient temperature (ca. $25^{\circ} \mathrm{C}$ ).

Here, we note that these results are much better than the contact angle of PFA. As typically reported by Takahara and coworkers, ${ }^{8}$ an Rf-containing polymer also obeys the SDA theory, and $\mathrm{R}_{\mathrm{f}} 5$ is too short to exhibit a fluorous property. In this sense, the present results using the SAM technique is remarkable.

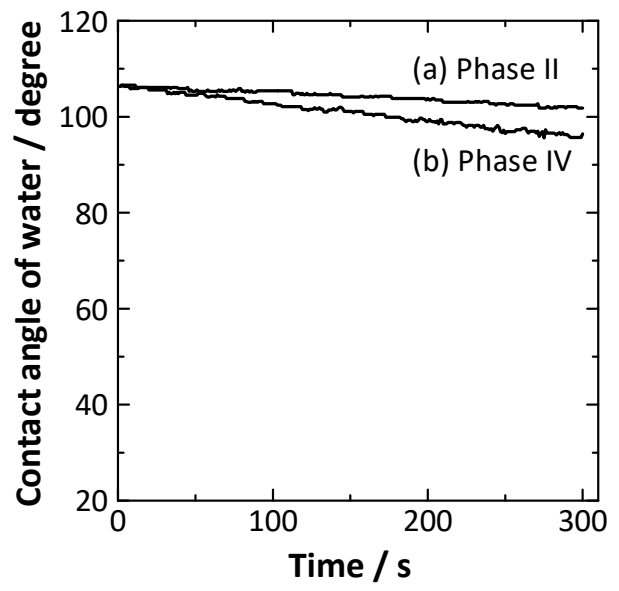

Figure 5. Time-dependent contact angle on the $\mathrm{R}_{\mathrm{f}} 5$-containing SAM measured at (a) $15^{\circ} \mathrm{C}$ and (b) $25^{\circ} \mathrm{C}$.

In addition, we have to pay another attention to the phase transition (Phase IV to II) at $19^{\circ} \mathrm{C}$. Above this temperature, the helical pitch about the $\mathrm{R}_{\mathrm{f}}$ chain is a little bit loosen ${ }^{24-27}$ from the point group of $\mathrm{D}_{13}$ to that of $\mathrm{D}_{15} .^{27,28}$ As a result, temperature above $19^{\circ} \mathrm{C}$ is inappropriate for the molecular spontaneous aggregation, which is not good for exhibiting a fluorous property. In fact, the fluorous property of the $\mathrm{R}_{\mathrm{f}} 5$-containing PFA is drastically lost and the contact angle is less than $50^{\circ}$ at a temperature above $19^{\circ} \mathrm{C}$ (cf. Fig. 11 in the reference \#8).

On the Rf5-containing SAM, in our present study, the contact angle at $15^{\circ} \mathrm{C}$ keeps a high value (Figure $5 \mathrm{a}$ ), and the angle at $25^{\circ} \mathrm{C}$ still exhibits a larger value than $90^{\circ}$ (Figure $5 \mathrm{~b}$ ), which can be recognized as hydrophobic.

In this manner, by employing the SAM technique, the silicon surface has readily been made fluorous using a short- $\mathrm{R}_{\mathrm{f}}$ containing compound. On this concept, the conventional PFA technique proves to be also good, since the polymer main chain works as the substrate to make the side chains immobile, and the side chains are spontaneously aggregated. Regardless, the PFA technique relies only on the "spontaneous" aggregation, the length of $\mathrm{R}_{\mathrm{f}} 7$ is critically necessary, for which the SAM technique should be better.

\section{Conclusion}

To make the solid surface fluorous, two points must be satisfied: 1) the molecules are immobilized on the surface, and 2) the best use of the spontaneous aggregation property of $R_{f}$ groups should be made. In the present study, the SAM technique is found great for fulfilling the two points, on which the PFOX dilemma has readily been overcome.

To furnish a wide variety of material surfaces such as a textile on this concept, a tough coating technique of SAM being robust to repeat washing and UV irradiation should be developed as the next step of research.

\section{Acknowledgment}

This work was financially supported by Iketani Science and Technology Foundation (0301031-A). We are also supported by a Grant-in-Aid for Scientific Research (A) (No. 15H02185 (TH)) and Grant-in-Aid for Young Scientists (B) (No. 17K14502 (TS)) from the Japan Society for the Promotion of Science (JSPS), to whom their thanks are due.

\section{References}

1. M. P. Krafft, J. G. Riess, Chem. Rev. 2009, 109, 1714.

2. T. Hasegawa, Chem. Rec. 2017, 17, 903.

3. T. Hasegawa, Chem. Phys. Lett. 2015, 627, 64.

4. C. Bunn, E. Howells, Nature 1954, 174, 549.

5. T. Albrecht, H. Elben, R. Jaeger, M. Kimmig, R. Steiner, G. Strobl, B. Stühn, H. Schwickert, C. Ritter, J. Chem. Phys. 1991, 95, 2807.

6. T. Hasegawa, T. Shimoaka, N. Shioya, K. Morita, M. Sonoyama, T. Takagi, T. Kanamori, ChemPlusChem 2014, 79, 1421.

7. Y.-L. Lee, Colloids Surf. A: Physicochem. Eng. Aspects 1999, 155, 221.

8. K. Honda, M. Morita, H. Otsuka, A. Takahara, Macromolecules 2005, 38, 5699.

9. V. Barry, A. Winquist, K. Steenland, Env. Health Perspec. 2013, 121, 1313.

10. N. Terasawa, Y. Hayakawa, H. Fukaya, E. Hayashi, K. Kato, S. Fujii, H. Sawada, K. Li, J. Kyokane, Polymer 1998, 39, 5889.

11. T. Nishino, M. Meguro, K. Nakamae, Int. J. Adhesion Adhesives 1999, 19, 399.

12. R. Maoz, J. Sagiv, J. Colloid Interf. Sci. 1984,100, 465.

13. E. Frydman, H. Cohen, R. Maoz, J. Sagiv, Langmuir 1997, 13, 5089.

14. S. Norimoto, S. Morimine, T. Shimoaka, T. Hasegawa, Anal. Sci. 2013, 29, 979.

15. M. Miah, M. Shahabuddin, M. Karikomi, M. Salim, E. Nasuno, N. Kato, K. Iimura, Bull. Chem. Soc. Jpn. 2016,89, 203.

16. J. Iwasa, K. Kumazawa, K. Aoyama, H. Suzuki, S. Norimoto, T. Shimoaka, T. Hasegawa, J. Phys. Chem. C 2016, 120, 2807.

17. T. Konishi, K. Yamaguchi, Bull. Chem. Soc. Jpn. 2016, 89, 
424.

18. D. Janssen, R. Palma, S. Verlaak, P. Heremans, W. Dehaen, Thin Solid Films 2006, 515, 1433.

19. T. Hasegawa, S. Takeda, A. Kawaguchi, J. Umemura, Langmuir 1995, 11, 1236.

20. T. Nishino, M. Meguro, K. Nakamae, M. Matsushita, Y. Ueda, Langmuir 1999, 15, 4321.

21. H. Schwickert, G. Strobl, M. Kimmig, J. Chem. Phys. 1991, 95, 2800.

22. J. Als-Nielsen, D. Jacquemain, K. Kjaer, F. Leveiller, M. Lahav, L. Leiserowitz, Phys. Rep. 1994, 246, 251.

23. D. K. Owens, J. Appl. Polym. Sci. 1969, 13, 1741.

24. Flack, J. Polym. Sci. Part 2 Polym. Phys. 1972, 10, 1799.

25. C.-K. Wu, M. Nicol, Chem. Phys. Lett. 1973, 21, 153.

26. R. K. Eby, E. S. Clark, B. L. Farmer, G. J. Piermarini, S. Block, Polymer 1990, 31, 2227.

27. J. F. Rabolt, G. Piermarini, S. Block, J. Chem. Phys. 1978, 69, 2872.

28. T. Shimoaka, M. Sonoyama, H. Amii, T. Takagi, T. Kanamori, T. Hasegawa, J. Phys. Chem. A 2017, 121, 8425 . 